\title{
Quantification of Structural Heterogeneities and Morphologies in Ultrathin Au-based Nanowire Systems using 4D STEM and Electron Tomography
}

\author{
Alexandra Bruefach ${ }^{1}$, Audrey Von Raesfeld ${ }^{1}$ and Mary Scott ${ }^{2}$ \\ ${ }^{1}$ Materials Science and Engineering, University of California, Berkeley, United States, ${ }^{2} \mathrm{UC}$ Berkeley, \\ Berkeley, California, United States
}

Hierarchical nanomaterial design has led to advances in numerous fields, including medicine, optics, and catalysis [1,2,3]. Local crystallographic structures and morphologies govern these behaviors, thus warranting deep investigation into how these structural variations impact these properties. Fourdimensional scanning transmission electron microscopy (4D-STEM) provides a unique opportunity to probe local crystal structure in diffraction space. This technique has the ability to provide a wealth of information on materials with complex arrangements.

There have been several reports of metallic nanowires taking on complex structures based on the BoerdijkCoxeter-Bernal (BCB) helix [4,5,6]. These structures are comprised of multiple tetrahedral units packed on their faces into an aperiodic helical structure. Most notably is the ultrathin ( 2nm) Au-Ag based system that rapidly twists into double helices upon induction with $\mathrm{Pd}, \mathrm{Pt}$, or $\mathrm{Au}$ salt [7]. These $\mathrm{Au}-\mathrm{Ag}$ seed structures are purported to exhibit a double-stranded BCB helical lattice, which shows indicative fan-like contrast in EM imaging due to the presence of icosahedral packing [4,7]. Initially, it was proposed that this material exhibits this mechanical response due to a lattice transformation from the BCB-type structure to FCC, caused by the strain buildup of interfacial Pd deposition [7]. Recent reports show crystalline and polycrystalline FCC Au-based nanowires with similar diameters also twist into double helices upon induction with $\mathrm{Ag}$ or $\mathrm{Pd}$ salt [6,7]. Although the behavior is similar, these systems were proposed to twist due to alloying effects leading to a lattice transformation towards the BCB-type structure. This intriguing behavior and argued reaction mechanisms across multiple ultrathin Au-based systems warrants further structural investigation. We demonstrate how this technique can be utilized to reveal population differences between morphologically similar structures.

However, 4D STEM alone does not provide insight on 3-dimensional (3D) morphologies of nanostructures, which is crucial for understanding catalytic behavior and unique optical behaviors, such as circular dichroism [8]. Instead, Electron Tomography (ET) provides necessary information regarding these 3D features such as curvature and surface morphology. Understanding these qualities is essential in understanding and interpreting the diffraction data, which is dependent on 3D structure.

We use statistical measurements from 4D STEM scans to understand crystallographic information that is not obtainable through nanoscale electron tomography, while recovering important morphological information such as curvature using nanoscale tomography. These two techniques, when interpreted collectively, provide a wealth of information revealing the crystal structure and morphology of the underlying twisted wire. These results allow for the mechanical transformation to be understood. 


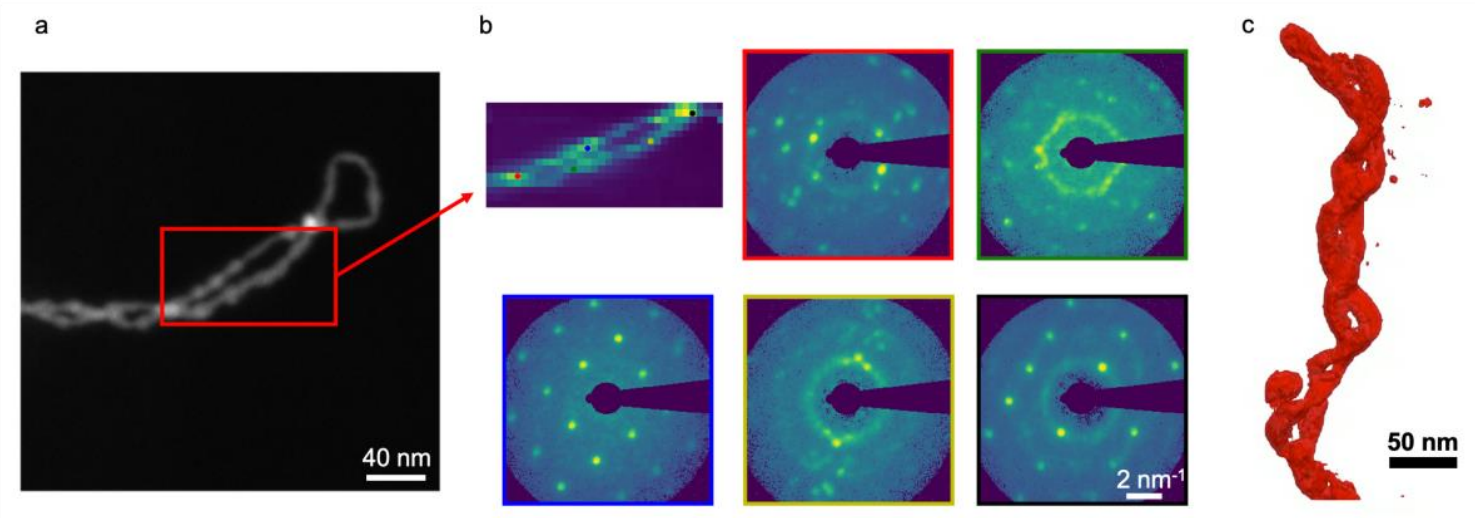

Figure 1. (a) STEM image of region for 4D STEM scan. (b) Virtual image and select diffraction patterns from 4D STEM scan. (c) Nanoscale tomography dataset.

\section{References}

1. Vimbela, Gina V., et al. "Antibacterial properties and toxicity from metallic nanomaterials." International journal of nanomedicine 12 (2017): 3941.

2. Link, Stephan, and Mostafa A. El-Sayed. "Optical properties and ultrafast dynamics of metallic nanocrystals." Annual review of physical chemistry 54.1 (2003): 331-366.

3. Astruc, Didier. "Transition-metal nanoparticles in catalysis: from historical background to the state-ofthe art." Nanoparticles and catalysis 16 (2008): 1-48.

4. Velázquez-Salazar, J. Jesús, et al. "Experimental evidence of icosahedral and decahedral packing in one-dimensional nanostructures." ACS nano 5.8 (2011): 6272-6278.

5. Zhu, Yihan, et al. "Experimental evidence of chiral gold nanowires with boerdijk-coxeter-bernal structure by atomic-resolution imaging." Microscopy and Microanalysis 20.S3 (2014): 1060-1061.

6. Mendoza-Cruz, Rubén, et al. "Helical growth of ultrathin gold-copper nanowires." Nano letters 16.3 (2016): 1568-1573.

7. Wang, Yong, et al. "Chiral transformation: from single nanowire to double helix." Journal of the American Chemical Society 133.50 (2011): 20060-20063.

8. Ophus, Colin. "Four-dimensional scanning transmission electron microscopy (4D-STEM): From scanning nanodiffraction to ptychography and beyond." Microscopy and Microanalysis 25.3 (2019): 563582. 